\title{
Volatile and Intravenous Anesthesia Alter Rat Liver Proteins: Proteomic Time Course Analysis of Rat Liver Proteins
}

\author{
Hisashi Watanabe ${ }^{1, *}$, Chihiro Kamagata ${ }^{2}$, Yoshiaki Tsuboko ${ }^{2}$ and Atsuhiro Sakamoto ${ }^{3}$ \\ ${ }^{l}$ Graduate Student, Department of Anesthesiology and Pain Medicine, Graduate School of Medicine, Nippon Medical \\ School, Tokyo, Japan \\ ${ }^{2}$ Staff Member, Department of Anesthesiology and Pain Medicine, Graduate School of Medicine, Nippon Medical \\ School, Tokyo, Japan \\ ${ }^{3}$ Professor and Chair, Department of Anesthesiology and Pain Medicine, Graduate School of Medicine, Nippon Medical \\ School, Tokyo, Japan
}

\begin{abstract}
Background: Our previous microarray study showed that sevoflurane anesthesia affects the expression of rat genes in multiple organs including the liver. In this study, we investigated whether liver protein expression was altered after propofol, sevoflurane, or isoflurane anesthesia. We also investigated differences in the time course of each drug 24 and $72 \mathrm{~h}$ after anesthesia.

Methods: Rats were randomly assigned to four groups (non-anesthetized group and three groups anesthetized at each time point, $\mathrm{n}=6$ per group). A venous catheter was inserted into the caudal vein of all rats. Rats were anesthetized with each agent for $6 \mathrm{~h}$, and the liver was obtained immediately after anesthesia. Proteomic analysis was performed.

Results: About 4200 spots in each gel were discriminated, and at least 2619 spots were matched. Using LC-MS/MS, we identified 47 spots for propofol, 45 spots for sevoflurane, and 21 spots for isoflurane that were differentially expressed (p < 0.05) $0 \mathrm{~h}$ after anesthesia. The numbers of altered proteins were 14 and 19 in the isoflurane and sevoflurane groups, respectively, $72 \mathrm{~h}$ after anesthesia, but alterations in 40 proteins were seen in the propofol group $72 \mathrm{~h}$ after anesthesia.

Conclusion: Volatile and intravenous anesthetics affected protein expression in the liver. Alterations were different for each drug, with isoflurane showing fewer altered proteins $0 \mathrm{~h}$ after anesthesia than the other two drugs. The time courses of those proteins were also different between individual anesthetics, suggesting fewer alterations in rat liver protein expression with volatile anesthetics than with propofol.
\end{abstract}

Keywords: Proteomics, anesthesia, liver, propofol, sevoflurane, isoflurane.

\section{INTRODUCTION}

Volatile anesthetics such as sevoflurane and isoflurane are widely used in surgery. Propofol, administered as an alkylphenol in a lipid emulsion [1], is also used in clinical surgery. These agents are metabolized in the liver [2,3]. Many studies have reported the effects of these anesthetics on the liver, including hepatotoxicity, hepatitis, organprotective effects via antioxidant proteins or enzymes, changes in lipid metabolism or serum lipid profiles, and others [4,5]. Hepatotoxic effects of anesthetics occur mainly due to hypersensitivity reactions, decreased hepatic blood flow, hypoxia, and free radical production $[4,5]$.

Hepatocellular protective functions of anesthetics through activation of antioxidant enzymes in the liver in an ischemic re-perfusion model were also reported [6,7]. Many

*Address correspondence to this author at the Department of Anesthesiology and Pain Medicine, Graduate School of Medicine, Nippon Medical School, 1-1-5 Sendagi, Bunkyo-ku, Tokyo 113-8603, Japan;

Tel: +81 35814 6243; Fax: +81 35685 3077; E-mail: s00-098@nms.ac.jp factors such as antioxidant enzymes, hepatic detox enzymes, heat shock proteins (HSPs), and direct effects of anesthetics may mediate hepatocellular protection from biological stresses $[7,8]$. Other hepatocellular antioxidant enzymes such as catalase (CAT), glutathione $\mathrm{S}$ transferase (GST), superoxide dismutase (SOD), and aldehyde dehydrogenase7A1 (ALDH7A1) also show liver protective functions by scavenging reactive oxygen species (ROS) or in other ways [9-13]. Intravenous anesthetics such as propofol do not seem to have comparable protective properties [14].

Statin therapy reduces the risk for perioperative cardiovascular events. HMG-CoA synthase synthesizes HMG-CoA from acetyl-CoA. HMG-CoA synthase is also a substrate for HMG-CoA reductase. HMG-CoA reductase converts HMG-CoA to mevalonate and is a main target of statins $[15,16]$. The process of HMG-CoA synthesis and conversion from HMG-CoA to mevalonate are well-known steps in cholesterol biosynthesis.

Apolipoprotein-A1 (Apo-A1) is a major protein component of high-density lipoprotein and plays an 
important role in maintaining cellular cholesterol homeostasis [17,18]. Monocytes lead to reduced Apo-A1 production in the liver by releasing cytokines during biological stress, and serum Apo-A1 levels are lowered by stress from major surgery, multiple trauma, and systemic inflammatory disease. Apo-A1 levels in the intensive care unit at admission are reported to be associated with morbidity [18].

Thus, there are many studies on liver proteins, but these proteins were assessed individually, and few reports have investigated the comprehensive influences of general anesthetics. Further, no reports have comprehensively evaluated alterations in liver protein expression due to anesthesia. Previously, we comprehensively showed changes in gene expression in multiple organs including the liver with sevoflurane anesthesia using microarray analysis [19]. Here, we undertook a comprehensive study to clarify the alterations in expression of rat liver proteins by general anesthetics such as propofol, isoflurane, and sevoflurane. We used proteomic analysis followed by liquid chromatographymass spectrometry (LC-MS) to analyze protein expression. This is the first comprehensive report to demonstrate alterations by general anesthetics at the protein level. The aim of our current study was to investigate whether general anesthetics alter rat liver protein expression and to compare differences in the time course of protein expression induced by each drug. We also investigated the relationship between the protein expression changes observed here and the gene expression changes we previously reported [19].

\section{MATERIALS AND METHODS}

\section{Animal Preparation}

Approval for this study and the procedures performed (notably the method of euthanasia) was obtained from the Animal Experimental Ethics Review Committee of Nippon Medical School (review number: 23-159). Nine-week-old male rats (specific pathogen-free Wistar rats; Saitama Experimental Animals) weighing $290 \pm 10 \mathrm{~g}$ were kept under temperature-controlled conditions on a 12:12-h light-dark cycle with free access to food and water.

\section{Administration of Anesthetic and Termination}

Rats were randomly assigned to four groups $(n=6$ per group). Rats undergoing volatile anesthesia were placed in a plastic box supplied with sevoflurane (Abbott Japan, Tokyo, Japan; 4.0\% gas-air mixture) [20] or isoflurane (Abbott Japan; 2.5\% gas-air mixture) [21], and normal saline was administered via a venous catheter $(1 \mathrm{~mL} / \mathrm{h})$. Rats undergoing intravenous anesthesia were housed in a plastic box and administered propofol (AstraZeneca, Osaka, Japan; $600 \mu \mathrm{g} / \mathrm{kg} / \mathrm{min}$ ) [22]. In the control group, rats were not given any anesthetics and were immediately sacrificed. Rats in the anesthetized groups (sevoflurane: group S-0, S-24, S72; isoflurane: group I-0, I-24, I-72; or propofol: group P-0, P-24, P-72) received anesthetics for $6 \mathrm{~h}$ and were killed by decapitation 0,24 , or $72 \mathrm{~h}$ after anesthesia. A venous catheter was inserted into the caudal vein of all rats in the anesthetized groups, and each rat was housed in an individual plastic box. All animals anesthetized with volatile or intravenous agents received $30 \%$ oxygen over the same time period and were allowed to breathe spontaneously.
Body temperature was maintained at a constant $37^{\circ} \mathrm{C}$. The left lateral lobe of the liver was removed from each rat within $3 \mathrm{~min}$ of death, immediately lysed in ISOGEN reagent (Nippon Gene, Tokyo, Japan), and frozen in liquid nitrogen. All liver samples were stored at $-80^{\circ} \mathrm{C}$ until proteome analysis.

\section{Physiological Parameters}

Physiological variables were measured in the following four groups not used for proteomic evaluation: a group treated with sevoflurane $(4.0 \%$ gas-air mixture), a group treated with isoflurane $(2.5 \%$ gas-air mixture), a group treated with propofol $(600 \mu \mathrm{g} / \mathrm{kg} / \mathrm{min})$, and a control (not anesthetized) group ( $\mathrm{n}=5$ in each group) [23]. A catheter was inserted into the tail vein of all rats, and normal saline was administered $(1 \mathrm{~mL} / \mathrm{h})$. During the experiment, each rat was maintained in the same conditions (body temperature, $\mathrm{O}_{2}$ supply, breathing, dose of anesthetics). We anesthetized the rats and cannulated the left femoral artery to measure heart rate and arterial blood pressure, and to draw blood samples for measuring arterial $\mathrm{PaO}_{2}$, arterial $\mathrm{PaCO}_{2}$, arterial blood $\mathrm{pH}$, and the plasma glucose concentration. After surgery, the rats were placed in a rat tunnel and allowed to recover from anesthesia. In the control group, blood samples were taken when awake. In the treated groups, anesthesia was maintained for $6 \mathrm{~h}$, and samples were taken before anesthetics were turned off. The experimental protocol is shown in Fig. (1).

\section{Two-dimensional Gel Electrophoresis}

We used the same differential in-gel electrophoresis (DIGE) design and protocol for two-dimensional gel electrophoresis (sample preparation, sample labeling, twodimensional electrophoresis) as in our previous study [24]. Each gel contained three different samples that were fluorescently labeled (CyDye DIGE Fluor Cy2, Cy3, and Cy5; GE Healthcare UK Ltd., Buckinghamshire, England) with the minimal labeling protocol [25]. First-dimension gel electrophoresis was performed using isoelectric focusing (IEF) gradient strip gels (24 cm pH 4-7) (GE Healthcare UK Ltd.) and an IPFphor IEF unit (GE Healthcare UK Ltd.). The second dimension, SDS-PAGE, was performed on $20 \mathrm{~cm} \times$ $24 \mathrm{~cm} 12.5 \%$ polyacrylamide gels. Electrophoresis was carried out using an Ettan DALTsix system (GE Healthcare UK Ltd.). Gels were scanned immediately using a Typhoon 9400 Variable Mode Imager (GE Healthcare UK Ltd.) with the same settings in all cases. The differential in-gel analysis module was used to identify protein spots and quantitatively compare the expression ratio of each individual protein spot feature between Cy5- and Cy3-labeled samples on a given gel after normalization to the Cy2 signal [26]. The biological variation analysis (BVA) module was used to collectively analyze individual matched gels.

\section{Spot Picking and In-gel Digestion}

Two-dimensional gels were stained with Deep Purple Total Protein Stain (GE Healthcare UK Ltd.). Proteins of interest were robotically excised in a 96-well plate format using Ettan Spot Picker and Digester workstations (GE Healthcare UK Ltd.). We then manually performed in-gel digestion as described [24]. 


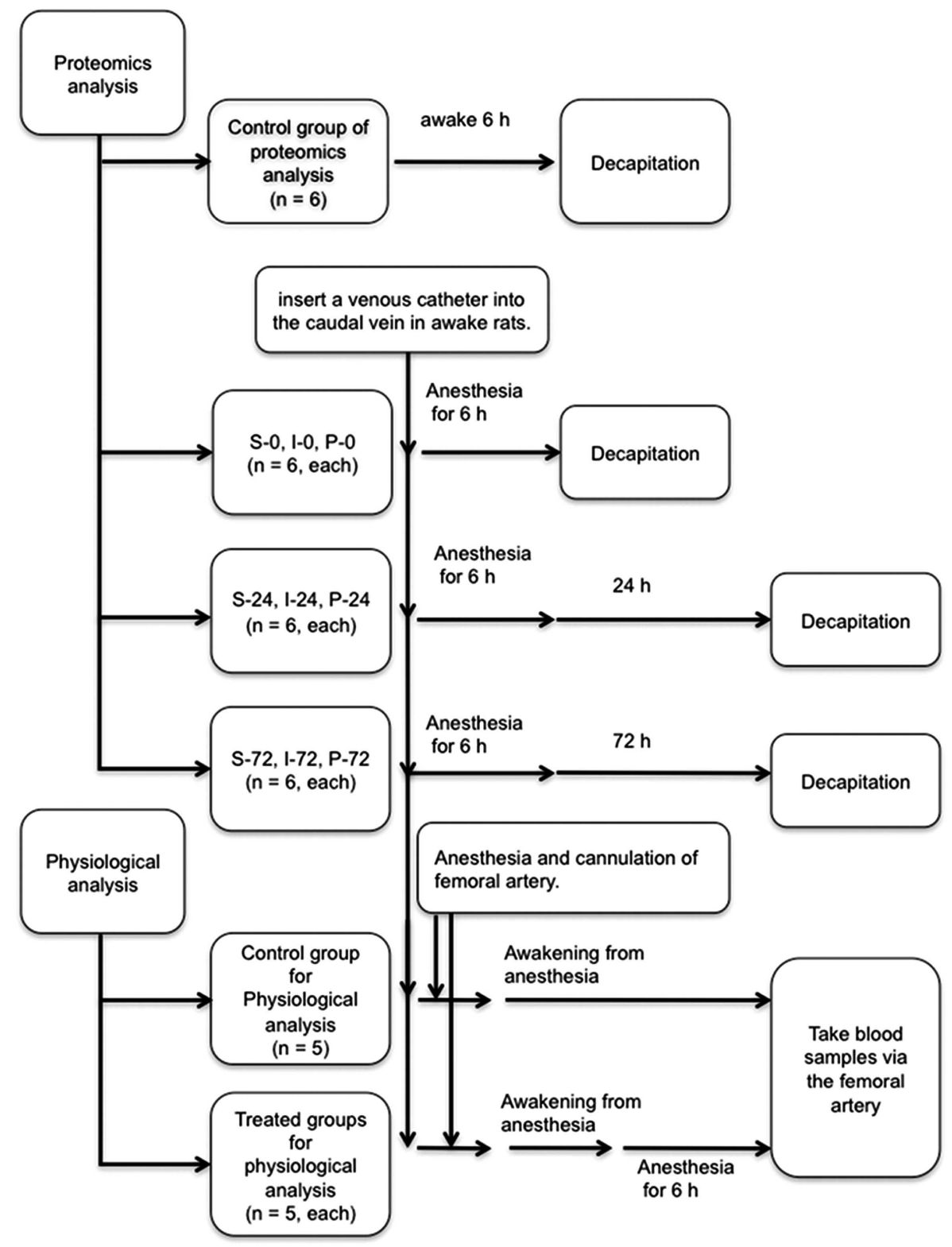

Fig. (1). Flow chart of the experimental protocols for physiological and proteomics analysis.

\section{Mass Spectroscopic Identification}

To investigate proteins that were changed during anesthesia, only proteins altered $0 \mathrm{~h}$ after anesthesia by at least one drug for mass spectroscopic identification were further examined 24 and $72 \mathrm{~h}$ after anesthesia. The resulting digested proteins were subjected to high-pressure liquid chromatography separation on a chromatography system (Paradigm Home 2009 R1, AMR Inc., Tokyo, Japan), which was coupled via an HTS-PAL (CTC Analytics, Zwingen, Switzerland) to an ion trap MS (amaZON ETD, Bruker Daltonics Inc., Billerica, MA, USA). The resulting MS and MS/MS spectra of peptide ions were searched using MASCOT software (www.matrix-science.com). The peptide species count is the number of peptides used for the MASCOT scoring report. Any MASCOT score $>70$ was considered significant. When multiple proteins were identified in a single spot, those with the highest numbers of peptides were considered to correspond to the spots. When multiple protein candidates were listed with an equal number of identified peptides, those with the higher MASCOT scores were selected.

\section{Statistical Analysis}

Data processing and analysis were performed with DeCyder v5.0 software (GE Healthcare Biosciences) [25]. We evaluated the matched spots with expression changes in spot volumes that were $>2.0$-fold and $\leq-2.0$-fold with oneway ANOVA; $P<0.05$ was considered significant. The mean expression ratio was obtained by comparing the expression value of the treated group to the mean value of the control group [27]. We examined each protein with Tukey's post-hoc test using the KyePlot v5.0 analysis software package (KyensLab Inc., Tokyo, Japan) to evaluate the significance of the association between the groups of all time points or all anesthetics. 
Table 1. Physiological Parameters in Control and Anesthetized Rats

\begin{tabular}{|l|l|l|l|l|}
\hline & Control & Propofol & Isoflurane & Sevoflurane \\
\hline \hline $\mathrm{pH}$ & $7.45 \pm 0.01$ & $7.40 \pm 0.04$ & $7.41 \pm 0.02$ & $7.44 \pm 0.02$ \\
\hline $\mathrm{PaO}_{2}$ & $109.1 \pm 6.9$ & $108 \pm 10.0$ & $99 \pm 7.8$ & $102 \pm 5.6$ \\
\hline $\mathrm{PaCO}_{2}$ & $36.9 \pm 1.4$ & $45.4 \pm 5.1$ & $41.2 \pm 4.2$ & $41.5 \pm 2.9$ \\
\hline $\mathrm{HR}$ & $311 \pm 25$ & $289 \pm 25$ & $308 \pm 25$ & $315 \pm 44$ \\
\hline $\mathrm{MAP}$ & $107 \pm 6.0$ & $95 \pm 17$ & $101 \pm 9$ & $96 \pm 8.5$ \\
\hline $\mathrm{GLU}$ & $123 \pm 27.0$ & $155 \pm 18.0$ & $141 \pm 12.9$ & $141 \pm 10.6$ \\
\hline
\end{tabular}

Data represent the means \pm standard deviation for five animals in each group. $\mathrm{PaO}_{2}$ : partial pressure of arterial oxygen (mmHg), $\mathrm{PaCO}$ : partial pressure of arterial carbon dioxide $(\mathrm{mmHg}), \mathrm{HR}$ : heart rate (beats/min), MAP: mean arterial pressure (mmHg), GLU: plasma glucose concentration (mg/dl). There were no significant differences in any of these parameters among the groups.

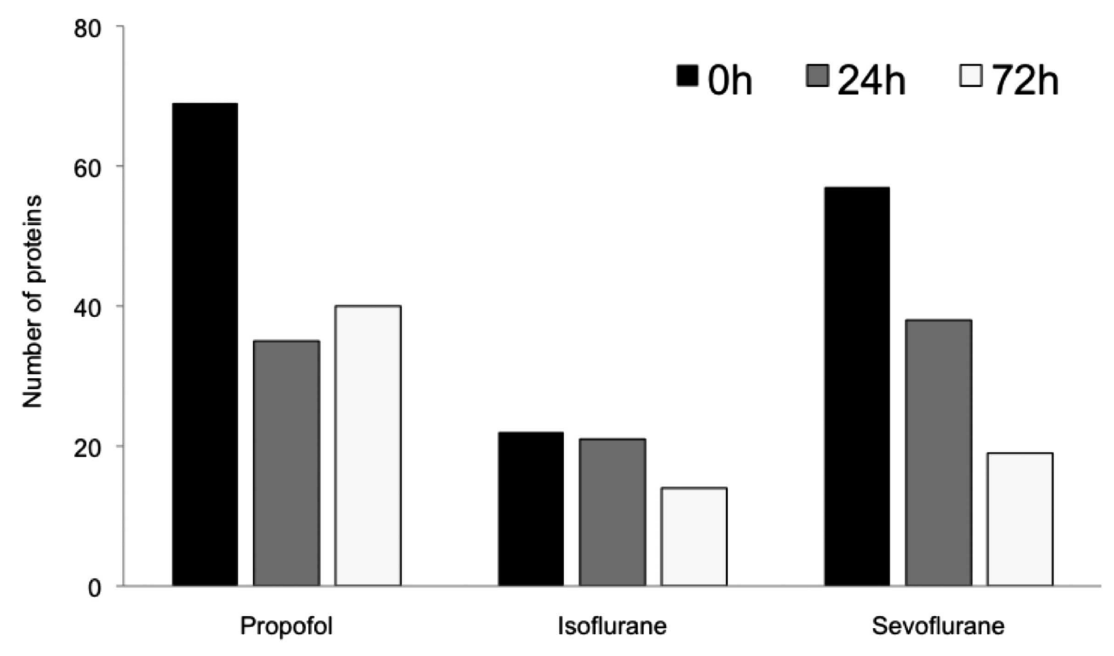

Fig. (2). Number of statistically significant, differentially expressed proteins (one-way ANOVA with Tukey's post-hoc test, $P<0.05$ ) at each time point. Fewer proteins were altered by isoflurane compared to propofol or sevoflurane immediately after anesthesia. Seventy-two hours after anesthesia, more proteins remained altered in the propofol group than in the isoflurane or sevoflurane groups.

\section{RESULTS}

All rats survived until sacrifice, and the data from all were used. There were no statistically significant differences in physiological parameters between the treated groups and the control group (Tukey's test, $P<0.05$ ) (Table 1).

Approximately 4200 spots were discriminated in each gel. In the anesthetized groups, at least 2619 matched spots were seen. Of these, 256 spots were identified with the BVA module, and 109 spots were differentially expressed with at least one drug $0 \mathrm{~h}$ after anesthesia. Of those 109 proteins, the numbers of spots differentially expressed were 69 in P-0, 35 in P-24, and 40 in P-72. In the isoflurane groups, the numbers of spots differentially expressed were 22 in I-0, 21 in I-24, and 14 in I-72. In the sevoflurane group, the numbers of spots differentially expressed were 57 in S-0, 38 in S-24, and 19 in S-72 (Fig. 2). All proteins were identified by LCMS/MS analysis, yielding 50 different proteins.

Based on their physiologic functions, these proteins were roughly subdivided into fundamental categories, including antioxidant proteins (antioxidant enzymes), HSPs or chaperones, cholesterol metabolism-related proteins, and other hepatocellular metabolism and homeostasis proteins. Proteins categorized as HSPs or chaperones were HSP70, HSP75, Dnaj homolog subfamily A member 3 precursor (HSP40), and protein disulfide isomerase (PDI) A4. HMGCoA synthase, Apo-A1, and estrogen sulfotransferase isoform 1 (EST-1) were categorized as cholesterol metabolismrelated proteins. CAT, $\mathrm{Cu}-\mathrm{Zn}$ SOD, ALDH7A1, ALDH2, sarcosine dehydrogenase, and GST-alpha3 were categorized as antioxidant enzymes. The protein name, pick spot no., MASCOT score, NCBI identifier, and the mean expression ratios at $0 \mathrm{~h}$ after anesthesia of all proteins are shown in Table 2.

Among these proteins, 10 were downregulated by all drugs immediately after anesthesia including Apo-A1, inner membrane protein mitochondrial isoform, nucleobinding 1 isoform 2, selenium binding protein precursor, vitamin D binding protein precursor, PDI, EST-1, neutral alphaglucosidase $\mathrm{AB}$, pyruvate kinase isozymes $\mathrm{R} / \mathrm{L}$ isoform, and HMG-CoA synthase. Some proteins downregulated by all agents $0 \mathrm{~h}$ after anesthesia returned to control levels 24 or 72 $\mathrm{h}$ after anesthesia, and some remained downregulated. The expression ratios of Apo-A1, HMG-CoA synthase, EST-1, 
Table 2. Identified Proteins

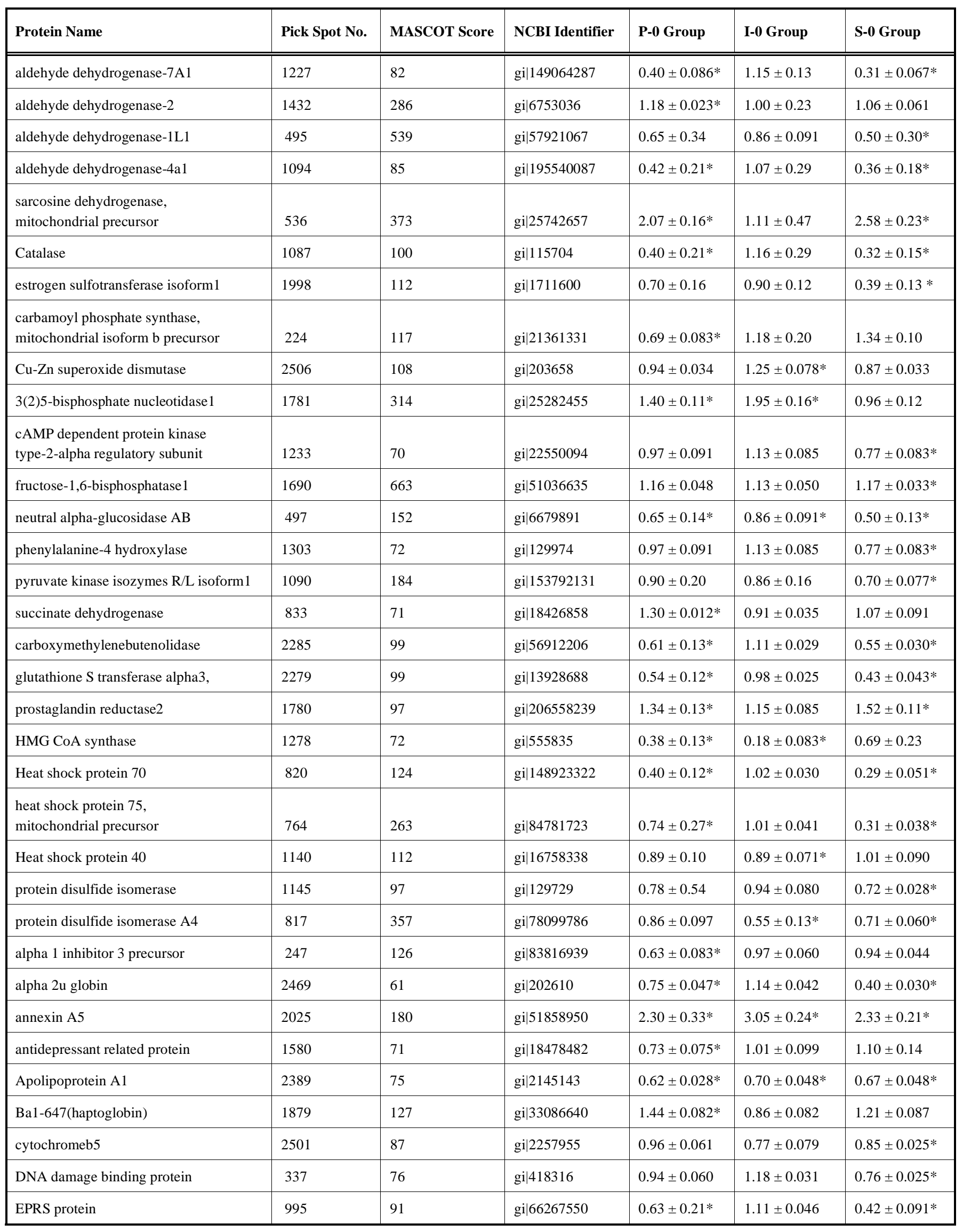


Table 2. contd...

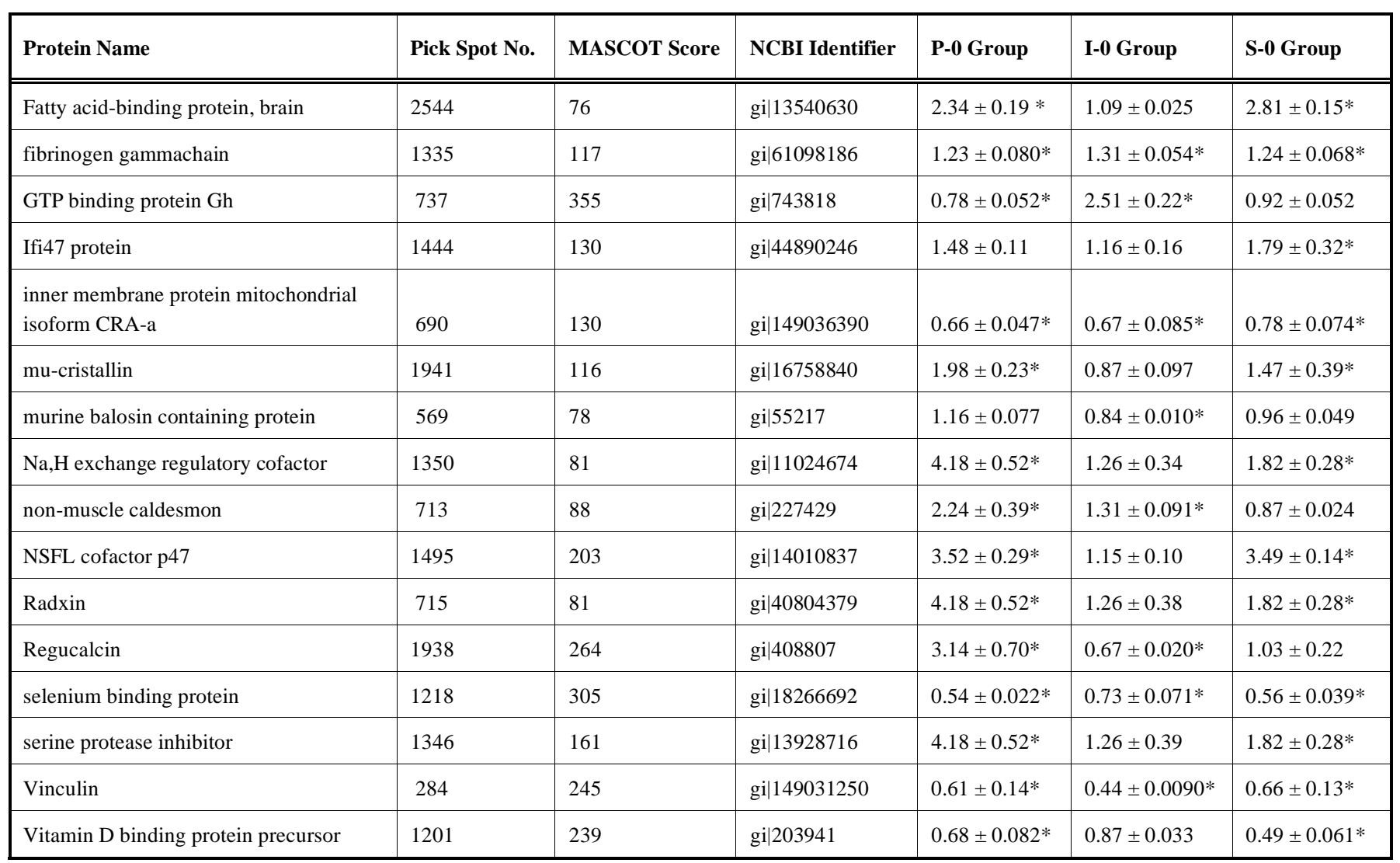

The values are the mean expression ratio \pm standard deviation. $(* P<0.05$ vs. control)

and vitamin D binding protein precursor were downregulated $0 \mathrm{~h}$ after anesthesia and remained low $72 \mathrm{~h}$ after anesthesia.

We identified another pattern in which the time courses of the propofol and sevoflurane groups were similar. ALDH1L1, ALDH4A1, ALDH7A1, CAT, EST-1, Cu-Zn SOD, PDI, GST alpha3, annexin A5, brain-type fatty acid binding protein, EPRS protein, alpha 1 inhibitor 3 precursor, DNA damage binding protein, GTP binding protein, fibrinogen gamma chain, murine globulin 1 precursor, nucleobinding1 isoform2, HSP70, and HSP75 fit this pattern. In this pattern, some proteins were categorized as antioxidant proteins. The expression ratio of many antioxidant proteins was also downregulated in P-0 and S-0, but the expression ratios of proteins in I-0 remained similar to control levels or were slightly upregulated. The time courses of some antioxidant proteins and cholesterol metabolism-related proteins in which we are interested are shown in Fig. (3).

\section{DISCUSSION}

Herein, we demonstrated expression changes in rat liver proteins induced by clinically used anesthetics such as sevoflurane, isoflurane, and propofol. We also showed differences in the time course of the expression ratio for each drug. Alterations were different for each drug, and we observed fewer alterations in rat liver protein expression with volatile anesthetics than with propofol at each time point. However, we were able to correlate few proteins induced by sevoflurane anesthesia with gene expression changes we had reported in our previous microarray study
[19]. The proteins we could consistently identify with gene expression in our previous study were HSP70, GST, and EST. Although gene expression changes do not necessarily correspond to protein production, we believe that the reasons we could not consistently identify other proteins and genes are as follows. First, there may be time delays needed for translation of the affected genes to proteins. In our current study, we aimed to identify proteins that were altered immediately after anesthesia; therefore, we did not investigate proteins at later time points if they were not altered $0 \mathrm{~h}$ after anesthesia. If we had investigated the proteins at later time points, we may have been able to identify other relationships between proteins and gene expression. Second, gene expression levels may not be correlated with protein expression levels, so we may have incorrectly excluded important proteins. Although the reasons we could not consistently identify other proteins and genes are unclear, our findings may suggest that some protein expression changes are related to gene expression changes. Further study will be needed to investigate this idea.

After $6 \mathrm{~h}$ of anesthesia, there were no significant differences in physiological parameters between the treated groups and the control group. Sufficient fluid infusion maintains hemodynamic stability in patients with no cardiovascular complications during general anesthesia. Further, positive pressure ventilation can easily increase the intrapleural pressure and affect the hemodynamic stability. We believe that hemodynamic stability was maintained in 


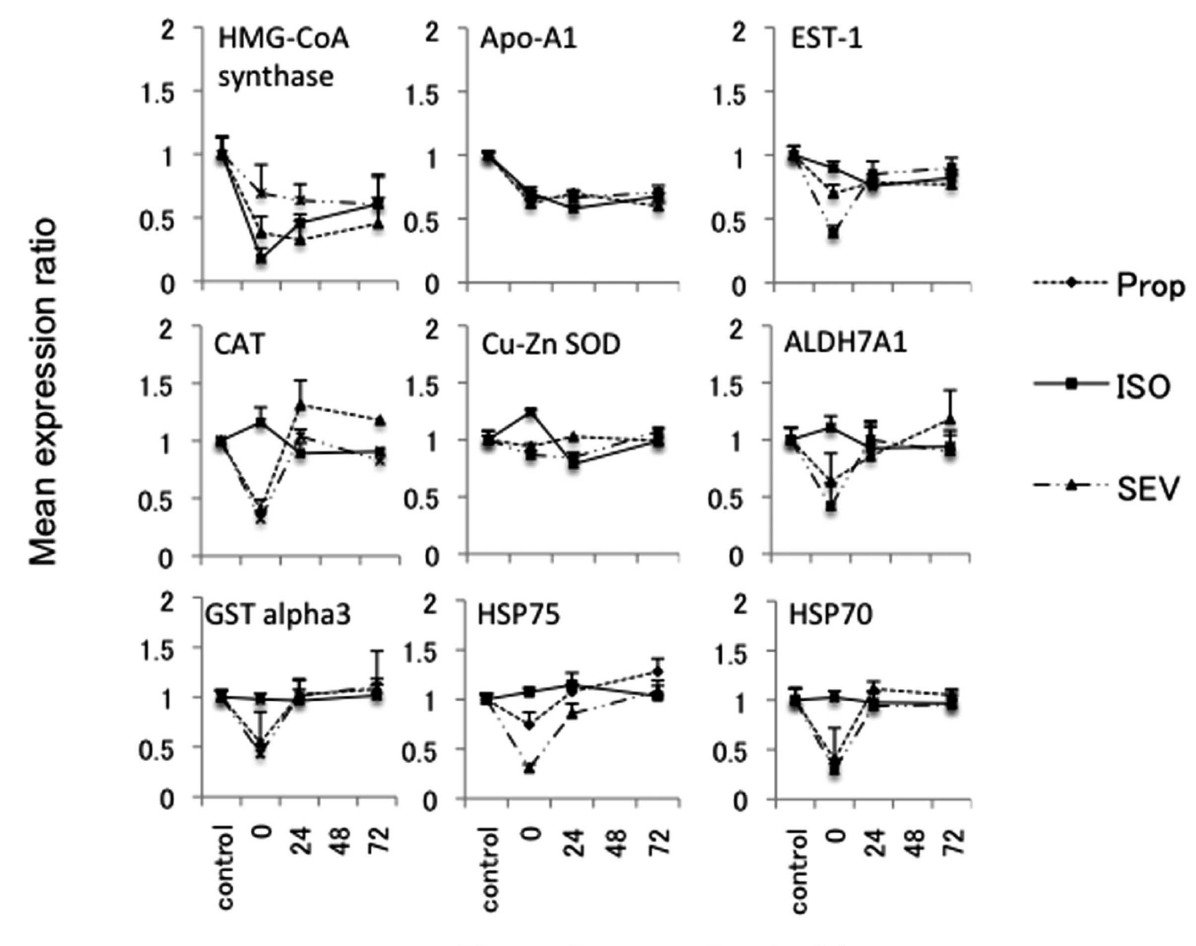

\section{Time after anesthesia ( $h$ )}

Fig. (3). The time course of proteins in which we were interested. Some proteins related to cholesterol metabolism, such as HMG-CoA synthase, Apo-A1, and EST-1, were downregulated immediately after anesthesia. Interestingly, this group of proteins remained downregulated at $72 \mathrm{~h}$ after anesthesia. The time course of some other proteins, such as organ protective enzymes related to the antioxidant systems and heat shock proteins, were similar in the propofol group and sevoflurane group, and the expression of these proteins in the isoflurane group was different from that in the other two groups. The changes in expression of antioxidant proteins and heat shock proteins returned to control levels for each protein at 24 or $72 \mathrm{~h}$ after awaking from anesthesia.

this study because the rats were administered sufficient amounts of intravenous fluids and allowed to breathe spontaneously. Consequently, protein expression changes should reflect pharmacological effects of anesthetics that are not due to physiological parameters.

HMG-CoA synthase was downregulated by all three drugs $0 \mathrm{~h}$ after anesthesia. The expression ratios of HMGCoA remained significantly low 24 and $72 \mathrm{~h}$ after anesthesia (Fig. 3). Downregulation of HMG-CoA synthase may reduce HMG-CoA in hepatocytes and may reduce cholesterol synthesis. Lindenauer et al., suggested that the effect of short-term inactivation of the cholesterol metabolism pathway by statins, such as for 2 days, may alter postoperative lipid metabolism [28]. In our current study, significant downregulation of HMG-CoA synthase lasted until $72 \mathrm{~h}$ after anesthesia, and thus, general anesthesia itself may alter post-operative lipid metabolism.

We also found decreased Apo-A1 expression in the liver with all drugs and at all time points. Apo-A1 plays an important role in cholesterol metabolism, and serum Apo-A1 levels are known to decrease with surgical operations. Our findings suggest that general anesthesia itself alters lipid metabolism, and downregulation of Apo-A1 after surgery may be partially due to anesthetics.

EST-1 is an important enzyme in the deactivation pathway of estrogens, which are essential sex hormones for mammalian reproduction that circulate systemically and bind to the estrogen receptor. Sulfonated estrogens cannot bind or activate the estrogen receptor, and thus EST-1 plays a role in controlling the deactivation pathway of estrogen [29]. Estrogen is synthesized from cholesterol, and inactivation of the cholesterol synthesis pathway may therefore also alter estrogen synthesis and EST-1 regulation. Proteins such as those involved in estrogen or cholesterol metabolism are essential in mammals, and there may be major effects of volatile or intravenous anesthetics.

Propofol itself may alter lipid metabolism because it is formulated as a lipid emulsion, but there were few differences between the expression ratios of altered proteins by the three agents. Our findings suggested that general anesthetic agents may reduce lipid metabolism, and the reduction may not depend on the type of agent. Changes in the lipid profile have been reported in several acute inflammatory states [30] and post-operative states [31], but no reports have evaluated alterations in cholesterol metabolism by anesthetics. In this study, we did not investigate changes in serum lipid profiles over time, and thus could not show further alterations in lipid profiles by anesthetics. However, the results suggest the possibility of various alterations in lipid profiles during and after anesthesia.

The expression ratios of some antioxidant proteins or antioxidant-related proteins in $\mathrm{P}-0$ and $\mathrm{S}-0$ were similar. Oxygen-derived free radicals are produced during organ injury including in the liver, and ROS may be involved in oxidative tissue injury [11]. Although oxygen therapy is a widely performed procedure, including during general 
anesthesia, production of ROS, the superoxide anion $\mathrm{O}_{2}{ }^{-}$, hydrogen peroxide $\mathrm{H}_{2} \mathrm{O}_{2}$, and the hydroxyl radical $\mathrm{HO} \bullet$ from partially reduced $\mathrm{O}_{2}$ is an unavoidable consequence of oxygen therapy. During oxidative stress, the intracellular redox balance is disrupted with increased production and accumulation of ROS, which may lead to mitochondrial dysfunction and subsequent ATP production [32]. Such species are scavenged by the catalytic activities of $\mathrm{Cu}-\mathrm{Zn}$ SOD and CAT and non-enzymatically by GST [11]. Mitochondria are the major source of ROS and contain many antioxidant and detoxifying enzymes and proteins $[12,13,33,34]$. ALDH7A1 is located in mitochondria where it mainly metabolizes betaine aldehyde to betaine. ALDH7A1 was recently reported to protect hepatic cells from oxidative stress by metabolizing lipid peroxidation molecules $[9,10]$. In this study, expression of CAT, $\mathrm{Cu}-\mathrm{Zn}$ SOD, GST, and ALDH7A1 was significantly downregulated in $\mathrm{P}-0$ and $\mathrm{S}-0$, and their expression ratios returned to control levels $72 \mathrm{~h}$ after anesthesia. In contrast, the expression ratio of these proteins remained at control levels or was upregulated in I-0, and then returned to control levels $72 \mathrm{~h}$ after anesthesia. Thus, propofol or sevoflurane anesthesia may limit the liver protective effects of the antioxidant systems. Conversely, isoflurane anesthesia may activate antioxidant systems, or it may not inactivate the hepatoprotective pathway. Volatile anesthetics such as isoflurane and sevoflurane were reported to have pre-conditioning or protective effects on the liver in ischemic re-perfusion or liver surgery models, but there are no reports regarding the pre-conditioning effect of propofol injection on hepatic surgery [6,35-37]. In those studies, changes in expression of antioxidant proteins such as CAT, Cu-Zn SOD, and GST after anesthesia were not evaluated [36,38]. Antioxidant systems have not been sufficiently examined at molecular levels, and our findings suggest the presence of alterations in the liver protective effect of antioxidant proteins by general anesthetics.

There are some limitations to our study. First, we did not perform other assays such as western blotting to investigate the influences of anesthetic agents on individual proteins. We aimed to comprehensively investigate alterations caused by anesthetic agents, but further analysis with other assays will be necessary to further interpret our findings. The agents we used in this study may be another limitation. Propofol is an alkylphenol formulated in a liquid emulsion and metabolized in the liver. We chose the administration durations and the times for taking samples based on our previous studies [19,25,39]; however, liquid emulsion itself may affect lipid profiles during or after propofol anesthesia. In clinical situations, propofol is usually injected with a lipid emulsion. Further, we aimed to compare the differences in altered proteins among anesthetic agents used in clinical practice, so we did not investigate a possible control for propofol administration. Third, we did not investigate changes in serum lipid profiles or blood biochemical values over time. Although we found several proteins that were altered by anesthetic agents, we could not compare them to changes in serum profiles over time after anesthesia.

In conclusion, we show exhaustively for the first time changes in liver protein expression by general anesthetics such as sevoflurane, isoflurane, and propofol. We also compared expression of proteins and the time course of changes in the proteins we identified. General anesthetics altered many hepatic cellular proteins, and the alterations in some proteins lasted $72 \mathrm{~h}$ after anesthesia. Volatile anesthesia altered fewer proteins than propofol. The number of proteins that were still affected by anesthetics 24 and $72 \mathrm{~h}$ after anesthesia was larger in the propofol group than in the sevoflurane or isoflurane groups. Identifying altered proteins and the time course of each drug may aid the clinical choice of anesthetic agents in surgery, especially in patients who have liver function complications, who undergo hepatic surgery, or who are anesthetized several times in a short period.

\section{CONFLICT OF INTEREST}

Declared none.

\section{ACKNOWLEDGEMENTS}

We would especially like to thank Takashi Matsui and Taishi Yamashita for their excellent technical assistance and Miyuki Takatori for her technical advice.

\section{REFERENCES}

[1] Simons, P.J.; Cockshott, I.D.; Douglas, E.J.; Gordon, E.A.; Hopkins, K. Rowland, M. Disposition in male volunteers of a subanaesthetic intravenous dose of an oil in water emulsion of $14 \mathrm{C}$ propofol. Xenobiotica, 1988, 18, 429-440.

[2] Njoku, D.; Laster, M.J.; Gong, D.H.; Eger, E.I. 2nd; Reed, G.F.; Martin, J.L. Biotransformation of halothane, enflurane, isoflurane, and desflurane to trifluoroacetylated liver proteins: association between protein acylation and hepatic injury. Anesth. Analg., 1997, 84, 173-178.

[3] Guitton, J.; Buronfosse, T.; Desage, M.; Finois, J.P.; Perdrix, J.P.; Brazier, J.L.; Beaune, P. Possible involvement of multiple human cytochrome $\mathrm{P} 450$ isoforms in the liver metabolism of propofol. $\mathrm{Br}$. J. Anaesth., 1998, 80, 788-795.

[4] Elliott, R.H.; Strunin, L. Hepatotoxicity of volatile anaesthetics. $\mathrm{Br}$ J. Anaesth., 1993, 70, 339-348.

[5] Naziroglu, M.; Gunay, C. The levels of some antioxidant vitamins, glutathione peroxidase and lipoperoxidase during the anaesthesia of dogs. Cell Biochem. Funct., 1999, 17, 207-212.

[6] Beck-Schimmer, B.; Breitenstein, S.; Urech, S.; De-Conno, E.; Wittlinger, M.; Puhan, M.; Jochum, W.; Spahn, D.R.; Graf, R., Clavien, P.A. A randomized controlled trial on pharmacological preconditioning in liver surgery using a volatile anesthetic. Ann. Surg., 2008, 248, 909-918.

[7] Schmidt, R.; Tritschler, E.; Hoetzel, A.; Loop, T.; Humar, M.; Halverscheid, L.; Geiger, K.K.; Pannen, B.H. Heme oxygenase-1 induction by the clinically used anesthetic isoflurane protects rat livers from ischemia/reperfusion injury. Ann. Surg., 2007, 245, 931-942.

[8] Hoetzel, A.; Leitz, D.; Schmidt, R.; Trischler, E.; Bauer, I.; Loop, T.; Humar, M.; Geiger, K.K.; Pannen, B.H. Mechanism of hepatic heme oxygenase-1 induction by isoflurane. Anesthesiology, 2006, 104, 101-109.

[9] Brocker, C.; Lassen, N.; Estey, T.; Pappa, A.; Cantore, M.; Oriova, V.V.; Chavakis, T.; Kabanagh, K.L.; Oppermann, U.; Vasiliou, V. Aldehyde dehydrogenase 7A1 (ALDH7A1) is a novel enzyme involved in cellular defense against hyperosmotic stress. J. Biol. Chem., 2010, 285, 18452-18463.

[10] Brocker, C.; Cantore, M.; Failli, P.; Vasiliou V. Aldehyde dehydrogenase 7A1 (ALDH7A1) attenuates reactive aldehyde and oxidative stress induced cytotoxicity. Chem. Biol. Interact., 2011, 191, 269-277.

[11] Hou, L.; Xie, K.; Qin, M.; Peng, D.; Ma, S.; Shang, L.; Li, N.; Li, S.; Ji, G.; Lu, Y.; Xiong, L. Effects of reactive oxygen species scavenger on the protective action of $100 \%$ oxygen treatment against sterile inflammation in mice. Shock, 2010, 33, 646-654.

[12] Mari, M.; Morales, A.; Colell, A.; Garcia-Ruiz, C.; FernandezCheca, J.C. Mitochondrial glutathione, a key survival antioxidant. Antioxid. Redox Signal., 2009, 11, 2685-2700. 
[13] Gallagher, E.P.; Gardner, J.L.; Barber, D.S. Several glutathione Stransferase isozymes that protect against oxidative injury are expressed in human liver mitochondria. Biochem. Pharmacol., 2006, 71, 1619-1628.

[14] Cope, D.K.; Impastato, W.K.; Cohen, M.V.; Downey, J.M. Volatile anesthetics protect the ischemic rabbit myocardium from infarction. Anesthesiology, 1997, 86, 699-709.

[15] Skrlin, S.; Hou, V. A review of perioperative statin therapy for noncardiac surgery. Semin. Cardiothorac. Vasc. Anesth., 2010, 14, 283-290.

[16] Biccard, B.M.; Sear, J.W.; Foex, P. Statin therapy: a potentially useful peri-operative intervention in patients with cardiovascular disease. Anaesthesia, 2005, 60, 1106-1114.

[17] Kobayashi, M.; Gouda, K.; Chisaki, I.; Ochiai, M.; Itagaki, S.; Iseki, K. Regulation mechanism of ABCA1 expression by statins in hepatocytes. Eur. J. Pharmacol., 2011, 662, 9-14.

[18] Chenaud, C.; Merlani, PG.; Roux-Lombard, P.; Burger, D.; Harbarth, S.; Luyasu, S.; Graf, J.D.; Dayer, J.M.; Ricou, B. Low apolipoprotein A-I level at intensive care unit admission and systemic inflammatory response syndrome exacerbation. Crit. Care. Med., 2004, 32, 632-637.

[19] Sakamoto, A.; Imai, J.; Nishikawa, A.; Honma, R.; Ito, E.; Yanagisawa, Y.; Kawamura, M.; Ogawa R.; Watanabe, S. Influence of inhalation anesthesia assessed by comprehensive gene expression profiling. Gene, 2005, 356, 39-48.

[20] Obal, D.; Preckel, B.; Scharbatke, H.; Müllenheim, J.; Höterkes, F.; Thämer, V.; Schlack, W. One MAC of sevoflurane provides protection against reperfusion injury in the rat heart in vivo. Br. J. Anaesth., 2001, 87, 905-911.

[21] Shichino, T.; Murakawa, M.; Adachi, T.; Arai, T.; Miyazaki, Y.; Mori, K. Effects of inhalation anaesthetics on the release of acetylcholine in the rat cerebral cortex in vivo. Br. J. Anaesth., 1998, $80,365-370$.

[22] Orth, M.; Barter, L.; Dominguez, C.; Atherley, R.; Carstens, E.; Antognini, J.F. Halothane and propofol differentially affect electroencephalographic responses to noxious stimulation. $B r . J$. Anaesth., 2005, 95, 477-484.

[23] Kawaguchi, H.; Hirakawa, K.; Miyauchi, K.; Koike, K.; Ohno, Y.; Sakamoto, A. Pattern recognition analysis of proton nuclear magnetic resonance spectra of brain tissue extracts from rats anesthetized with propofol or isoflurane. PLoS. One, 2010, 5, e11172.

[24] Kamagata, C.; Tsuboko, Y.; Okabe, T.; Sato, C.; Sakamoto, A. Proteomic analysis of rat brains in a model of neuropathic pain following exposure to electroconvulsive stimulation. Biomed. Res., 2011, 32, 91-102.

[25] Tsuboko, Y.; Sakamoto, A. Propofol anaesthesia alters the cerebral proteome differently from sevoflurane anaesthesia. Biomed. Res., 2011, 32, 55-65.
[26] Marouga, R.; David, S.; Hawkins, E. The development of the DIGE system: 2D fluorescence difference gel analysis technology. Anal. Bioanal. Chem., 2005, 382, 669-678.

[27] Okano, T.; Kondo, T.; Kakisaka, T.; Fujii, K.; Yamada, M.; Kato, H.; Nishimura, T.; Gemma, A.; Kudoh, S.; Hirohashi, S. Plasma proteomics of lung cancer by a linkage of multi-dimensional liquid chromatography and two-dimensional difference gel electrophoresis. Proteomics, 2006, 6, 3938-3948.

[28] Lindenauer, P.K.; Pekow, P.; Wang, K.; Gutierrez, B.; Benjamin, E.M. Lipid-lowering therapy and in-hospital mortality following major noncardiac surgery. JAMA, 2004, 291, 2092-2099.

[29] Gong, H.; Jarzynka, M.J.; Cole, T.J.; Lee, J.H.; Wada, T.; Zhang, B.; Gao, J.; Song, W.C.; De-Franco, D.B.; Cheng, S.Y.; Xie, W. Glucocorticoids antagonize estrogens by glucocorticoid receptormediated activation of estrogen sulfotransferase. Cancer Res., 2008, 68, 7386-7393.

[30] Alvarez, C.; Ramos, A. Lipids, lipoproteins, and apoproteins in serum during infection. Clin. Chem., 1986, 32, 142-145.

[31] Canivet, J.L.; Damas, P.; Buret, J.; Lamy, M. Postoperative changes in lipid profile: their relations with inflammatory markers and endocrine mediators. Acta. Anaesthesiol. Belg., 1989, 40, 263-268.

[32] Marin-Garcia, J.; Goldenthal, M.J. Mitochondrial centrality in heart failure. Heart Fail. Rev., 2008, 13, 137-150.

[33] Cao, Y.; Kennedy, R.; Klimberg, V.S. Glutamine protects against doxorubicin-induced cardiotoxicity. J. Surg. Res., 1999, 85, 178-182.

[34] Hayes, J.D.; McLellan, L.I. Glutathione and glutathione-dependent enzymes represent a co-ordinately regulated defence against oxidative stress. Free Radic. Res., 1999, 31, 273-300.

[35] Tao, K.M.; Yang, L.Q.; Liu, Y.T.; Tao, Y.; Song, J.C.; Wu, F.X.; $\mathrm{Yu}$, W.F. Volatile anesthetics might be more beneficial than propofol for postoperative liver function in cirrhotic patients receiving hepatectomy. Med. Hypotheses, 2010, 75, 555-557.

[36] Imai, M.; Kon, S.; Inaba, H. Effects of halothane, isoflurane and sevoflurane on ischemia-reperfusion injury in the perfused liver of fasted rats. Acta. Anaesthesiol. Scand., 1996, 40, 1242-1248.

[37] Bedirli, N.; Ofluoglu, E.; Kerem, M.; Utebey, G.; Alper, M.; Yilmazer, D.; Bedirli, A.; Ozlu, O.; Pasaogle, H. Hepatic energy metabolism and the differential protective effects of sevoflurane and isoflurane anesthesia in a rat hepatic ischemia-reperfusion injury model. Anesth. Analg.; 2008, 106, 830-837.

[38] Ishida, H.; Kadota, Y.; Sameshima, T.; Nishiyama, A.; Oda, T.; Kanmura, Y. Comparison between sevoflurane and isoflurane anesthesia in pig hepatic ischemia-reperfusion injury. J. Anesth., 2002, 16, 44-50.

[39] Nakazato, K.; Yoshida, Y.; Takemori, K.; Kobayashi, K.; Sakamoto, A. Expressions of genes encoding drug-metabolizing enzymes are altered after sevoflurane, isoflurane, propofol or dexmedetomidine anesthesia. Biomed. Res., 2009, 30, 17-24.

() Watanabe et al.; Licensee Bentham Open.

This is an open access article licensed under the terms of the Creative Commons Attribution Non-Commercial License (http://creativecommons.org/licenses/ by-nc/3.0/) which permits unrestricted, non-commercial use, distribution and reproduction in any medium, provided the work is properly cited. 